\title{
The development of bronchiectasis on chest computed tomography in children with cystic fibrosis: can pre-stages be identified?
}

\author{
Leonie A. Tepper ${ }^{1,2} \cdot$ Daan Caudri $^{1}$ - Adria Perez Rovira ${ }^{1,3}$. \\ Harm A. W. M. Tiddens ${ }^{1,2,4} \cdot$ Marleen de Bruijne (3,5 $^{3}$
}

Received: 25 June 2015 /Revised: 8 March 2016 / Accepted: 11 March 2016 /Published online: 23 April 2016

(C) The Author(s) 2016. This article is published with open access at Springerlink.com

\begin{abstract}
Objective Bronchiectasis is an important component of cystic fibrosis (CF) lung disease but little is known about its development. We aimed to study the development of bronchiectasis and identify determinants for rapid progression of bronchiectasis on chest $\mathrm{CT}$.

Methods Forty-three patients with CF with at least four consecutive biennial volumetric CTs were included. Areas with bronchiectasis on the most recent $\mathrm{CT}$ were marked as regions of interest (ROIs). These ROIs were generated on all preceding CTs using deformable image registration. Observers indicated whether: bronchiectasis, mucus plugging, airway wall thickening, atelectasis/consolidation or normal airways were present in the ROIs.
\end{abstract}

Electronic supplementary material The online version of this article (doi:10.1007/s00330-016-4329-z) contains supplementary material, which is available to authorized users.

Harm A. W. M. Tiddens

h.tiddens@erasmusmc.nl

1 Department of Pediatric Pulmonology, Erasmus MC, Sophia Children's Hospital, Rotterdam, The Netherlands

2 Department of Radiology, Erasmus MC, Sophia Children's Hospital, Rotterdam, The Netherlands

3 Biomedical Imaging Group Rotterdam, Departments of Radiology and Medical Informatics, Erasmus MC, Rotterdam, The Netherlands

4 Department of Pediatric Pulmonology and Radiology, Erasmus Medical Center, Sophia Children's Hospital, Dr. Molewaterplein 60, room SP-3464, 3015 GJ Rotterdam, The Netherlands

5 Department of Computer Science, University of Copenhagen, Copenhagen, Denmark
Results We identified 362 ROIs on the most recent CT. In 187 (51.7\%) ROIs bronchiectasis was present on all preceding CTs, while 175 ROIs showed development of bronchiectasis. In 139/175 (79.4\%) no pre-stages of bronchiectasis were identified. In 36/175 (20.6\%) bronchiectatic airways the following pre-stages were identified: mucus plugging $(17.7 \%)$, airway wall thickening $(1.7 \%)$ or atelectasis/consolidation $(1.1 \%)$. Pancreatic insufficiency was more prevalent in the rapid progressors compared to the slow progressors $(\mathrm{p}=0.05)$. Conclusion Most bronchiectatic airways developed within 2 years without visible pre-stages, underlining the treacherous nature of $\mathrm{CF}$ lung disease. Mucus plugging was the most frequent pre-stage.

Key Points

- Development of bronchiectasis in cystic fibrosis lung disease on CT.

- Most bronchiectatic airways developed within 2 years without pre-stages.

- The most frequently identified pre-stage was mucus plugging.

- This study underlines the treacherous nature of CF lung disease.

Keywords Cystic fibrosis $\cdot$ Paediatrics $\cdot$ High resolution computed tomography $\cdot$ Bronchiectasis $\cdot$ Lung disease
Abbreviations
CF Cystic fibrosis
CT Computed tomography
$\mathrm{FEV}_{1} \quad$ Forced expiratory volume in $1 \mathrm{~s}$
FVC Forced vital capacity
ROI Region of interest 


\section{Introduction}

An important component of cystic fibrosis (CF) lung disease is bronchiectasis [1]. Bronchiectasis is a permanent and irreversible abnormal dilatation of the bronchial lumen and is most reliably detected by chest computed tomography (CT) [2-5].

Bronchiectasis has been observed on chest $\mathrm{CT}$ in infants with $\mathrm{CF}$ as young as 10 weeks of age [6-11]. At 3-5 years of age, $50-70 \%$ of children with CF already have bronchiectasis, which contributes importantly to the morbidity and mortality in CF $[6,8,12]$. To prevent the development of bronchiectasis it is of great clinical importance to identify early prestages on CT with the aim of preventing further progression. Mucus plugging, airway wall thickening and atelectasis or consolidation have been observed to develop early in life in many patients and these changes could potentially be prestages for the development of bronchiectasis [1]. Studies showed that once bronchiectatic airways are present, they progress in severity $[1,6,10,13,14]$. However, it remains unclear how bronchiectatic airways evolve and why progression is more rapid in some patients [15].

Therefore, this study aims to identify pre-stages of bronchiectasis in $\mathrm{CF}$ on chest $\mathrm{CT}$ and to determine which patients are at risk for a rapid progression of bronchiectasis.

\section{Methods}

\section{Study population}

This retrospective study used longitudinally collected clinical data from the routine annual evaluation of CF patients under treatment in the Erasmus MC-CF Center (Rotterdam, The Netherlands) from January 2005-May 2013. We included 43 clinically stable CF patients who had at least four consecutive volumetric inspiratory CTs to guarantee a minimum follow-up period of 6 years. A routine chest $\mathrm{CT}$ is performed biennially as part of the annual evaluation programme. During this study period the annual evaluation programme was changed to structure the follow-up schedule for routine chest CTs, so that every child with CF has a CT at the same age $(6,8,10,12,14,16,18$ years).

Patients who had a lung transplant in the study period were excluded. Furthermore, patients with a pulmonary exacerbation or pulmonary complications (e.g. pneumothorax or haemoptysis) at the time of CT scanning were excluded. A pulmonary exacerbation was defined as receiving intravenous antibiotics for respiratory symptoms.

We denoted the most recent available volumetric CT made during annual evaluation as $\mathrm{CT}_{\text {baseline. }} \mathrm{CT}_{\text {minus2,4,6,8 }}$ are denoted as respectively the CTs made $2,4,6$ or 8 years prior to $\mathrm{CT}_{\text {baseline. }}$
This study was approved by the Institutional Review Board of the Erasmus MC-CF Center (MEC-2013-593).

\section{CT acquisition and spirometry}

CTs were performed on different CT scanners using different low-dose protocols. Most CTs $(77.8 \%)$ were predominantly executed on the Emotion 6 (Siemens Emotion 6, Siemens Healthcare, Germany). Since 2010 the remainder of the scans were made on a new CT scanner (SOMATOM Definition Flash, Siemens Healthcare, Germany). More details about the CT protocols and CT scanners are given in the on-line supplementary material (e-Table 1).

From 2007 onwards, most volumetric CTs were spirometer controlled. Spirometer-controlled CT scanning was introduced in our hospital to optimize inspiratory and expiratory volume. If due to logistic reasons $\mathrm{CT}$ was not spirometer controlled, then training for breath holds prior to the scan and instructions during the scan were given by the lung function technician (on-line supplementary material).

Spirometry was performed at the annual evaluation using a diagnostic system (Jaeger AG, Würzburg, Germany). The spirometry parameters included for analysis were forced expiratory volume in $1 \mathrm{~s}\left(\mathrm{FEV}_{1}\right)$ and forced vital capacity (FVC). $\mathrm{FEV}_{1}$ and $\mathrm{FVC}$ were expressed as a percentage of predictive values, calculated using the Stanojevic et al. reference equations [16].

\section{CT analysis}

According to the definition of the validated CF-CT scoring system [17-19], bronchiectasis is present if the bronchial lumen diameter is larger than the adjacent pulmonary artery outer diameter, or if there is a lack of tapering for at least $2 \mathrm{~cm}$ distal to a branching point. Based on literature about the pathophysiology of bronchiectasis and based on the expertise of a panel consisting of a paediatric pulmonologist (HT), a radiologist (PC), a biomedical imaging expert (MB) and a $\mathrm{PhD}$ student (LT), five mutually exclusive categories for classifying pre-stages of bronchiectasis were defined. These categories were: (1) bronchiectasis (bronchial lumen diameter is larger than the adjacent pulmonary artery outer diameter, or lack of tapering for at least $2 \mathrm{~cm}$, Fig. 1), (2) mucus plugging (filling of clearly identifiable bronchi, Fig. 1), (3) airway wall thickening (ratio between the bronchial wall thickness and the outer diameter of the adjacent pulmonary artery being more than $33 \%$, Fig. 1), (4) atelectasis or consolidation and (5) normal airways. These categories are well defined as part of the CF-CT scoring system and are further explained in the online supplementary material [17-19].

CTs were de-identified and randomized. One observer (observer 2: DZ) encircled all areas with bronchiectasis 
Table 1 Baseline characteristics of the study cohort

\begin{tabular}{|c|c|c|c|c|c|}
\hline Characteristic & $\mathrm{CT}_{\text {baseline }}$ & $\mathrm{CT}_{\text {minus } 2}$ & $\mathrm{CT}_{\text {minus }} 4$ & $\mathrm{CT}_{\text {minus }} 6$ & $\mathrm{CT}_{\text {minus } 8}$ \\
\hline Number of patients & 43 & 43 & 43 & 43 & 4 \\
\hline Number of regions of interest & 367 & 367 & 367 & 367 & 46 \\
\hline Gender (males) & $18(41.9)$ & $18(41.9)$ & $18(41.9)$ & $18(41.9)$ & $3(75.0)$ \\
\hline Age, year & $15.3(9-24)$ & $13.2(6-22)$ & $11.0(4-18)$ & $9.0(2-16)$ & $6.8(2-10)$ \\
\hline \multicolumn{6}{|l|}{ SES $^{\#}$} \\
\hline Low & $12(29.3)$ & $12(29.3)$ & $12(29.3)$ & $12(29.3)$ & $1(25.0)$ \\
\hline Average & $15(36.6)$ & $15(36.6)$ & $15(36.6)$ & $15(36.6)$ & $1(25.0)$ \\
\hline High & $13(31.7)$ & $13(31.7)$ & $13(31.7)$ & $13(31.7)$ & $2(50.0)$ \\
\hline Scientific & $1(2.4)$ & $1(2.4)$ & $1(2.4)$ & $1(2.4)$ & $0(0.0)$ \\
\hline \multicolumn{6}{|l|}{ Genetics } \\
\hline Homozygous dF508 & $30(69.8)$ & $30(69.8)$ & $30(69.8)$ & $30(69.8)$ & $3(75.0)$ \\
\hline Heterozygous dF508 & $9(20.9)$ & $9(20.9)$ & $9(20.9)$ & $9(20.9)$ & $1(25.0)$ \\
\hline Heterozygous other mutation & $4(9.3)$ & $4(9.3)$ & $4(9.3)$ & $4(9.3)$ & $0(0.0)$ \\
\hline \multicolumn{6}{|l|}{ Presence of co-morbidities } \\
\hline Pancreatic insufficient & $40(93.0)$ & $40(93.0)$ & $40(93.0)$ & $40(93.0)$ & $3(75.0)$ \\
\hline CFRD & $10(23.3)$ & $10(23.3)$ & $10(23.3)$ & $10(23.3)$ & $0(0.0)$ \\
\hline Asthma & $3(7.0)$ & $3(7.0)$ & $3(7.0)$ & $3(7.0)$ & $0(0.0)$ \\
\hline ABPA & $2(4.7)$ & $2(4.7)$ & $2(4.7)$ & $2(4.7)$ & $0(0.0)$ \\
\hline Chronic colonization $\mathrm{Pa}$ & $8(18.6)$ & $8(18.6)$ & $8(18.6)$ & $8(18.6)$ & $1(25.0)$ \\
\hline BMI & $19.2(15-36)$ & $18.0(14-30)$ & $17.0(5-31)$ & $16.5(14-25)$ & $15.7(15-17)$ \\
\hline $\mathrm{FEV}_{1}, \%$ predicted & $86.5(33-106)$ & $85.4(37-112)$ & $85.0(40-113)$ & $85.8(42-126)$ & $88.4(85-92)$ \\
\hline FVC, $\%$ predicted & $94.0(49-115)$ & $92.3(56-128)$ & $95.2(57-118)$ & $93.2(49-128)$ & $99.0(94-102)$ \\
\hline
\end{tabular}

Data are presented as no. (\%) or median (range)

$\mathrm{CT}_{\text {baseline-minus } 8}$ indicate the time points at which the $\mathrm{CTs}$ were made, with $\mathrm{CT}_{\text {baseline }}$ representing the most recent $\mathrm{CT}$ and $\mathrm{CT}_{\text {minus2-8 }}$ respectively representing the $\mathrm{CT}$ made $2,4,6$ or 8 years before $\mathrm{CT}_{\text {baseline }}$

\# SES: socio-economic status based on the highest level of education of either parent $\left(\mathrm{n}=41\right.$ at $\mathrm{CT}_{\text {baseline-minus6 }}$ and $\mathrm{n}=4$ at $\left.\mathrm{CT}_{\text {minus } 8}\right)$

\$ Indicating the number of patients having co-morbidities

$\$$ Chronic colonization $P a$ : defined as $\geq$ three consecutive positive respiratory cultures for Pseudomonas aeruginosa $(P a)$ from birth to $\mathrm{CT}_{\mathrm{baseline}}$ $C F R D$ CF-related diabetes, $A B P A$ allergic bronchopulmonary aspergillosis, $B M I$ body mass index, $F E V_{l}$ forced expiratory volume in $1 \mathrm{~s}, F V C$ forced vital capacity

(bronchial lumen diameter-pulmonary artery outer diameter ratio $>2$ or saccular bronchiectasis) in the most recent volumetric inspiratory $\mathrm{CT}\left(\mathrm{CT}_{\text {baseline }}\right)$. Those areas were marked as a region of interest $(\mathrm{ROI})$ using tools in our image analysis platform (®Myrian Onco XL, Intrasense, France). By using deformable image registration, the same areas were automatically identified in the previous $\mathrm{CTs}\left(\mathrm{CT}_{\text {minus2-minus8 }}\right)$. All preceding ROIs were assessed by two experienced observers with respectively 1 and 2 years of experience (HO and DZ) and assigned to one of the five above-mentioned categories. Category 2-5 reflects a potential pre-stage in the ROI of the bronchiectatic airway observed on the most recent $\mathrm{CT}$.

ROIs were assessed in random order with respect to patients and order of CT scans ( $\left.\mathrm{CT}_{\text {minus2-minus8 }}\right)$. Therefore, the observers had no knowledge of the assigned category of the ROIs in previous or later scans.

Fig. 1 Illustration of scoring categories: bronchiectasis (left), mucus plugging (middle) and airway wall thickening (right)

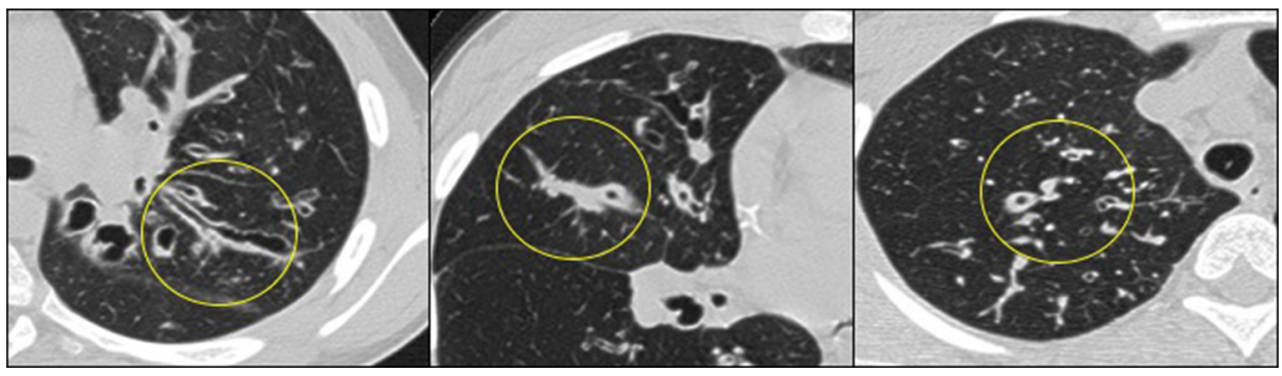




\section{Statistical analysis}

To assess observer agreement kappa scores were calculated. For the interobserver agreement, a random subset of 35 patients with a total of 1,230 ROIs were scored by two observers (H.O. and D.Z.). For the intra-observer agreement, a subset of 20 patients with 195 ROIs were rescored by H.O. after 1 month. In the final analyses, only the scores of observer H.O. were used. Although no universally accepted standards are available for what constitutes good agreement, kappa scores of $<0.40$, between 0.4 and 0.75 , and $\geq 0.75$ are considered to represent poor, moderate to good and excellent agreement, respectively [20].

Descriptive statistics were used to describe the CT findings.

We calculated how often bronchiectasis was persistent in all CTs and how often it was directly preceded by mucus plugging, airway wall thickening, atelectasis/consolidation, or normal airways.

In order to distinguish patients who rapidly developed bronchiectasis from previously normal airways from the ones who were likely to have a pre-stage, we created two progression groups: a rapid progression group and a slow progression group. The number of ROIs in which bronchiectasis was directly preceded by normal airways in any two successive scans was calculated. The median number of these rapidly progressing ROIs per patient was calculated (median $=3$ ) and used as the cut-off point to define the groups of rapid and slow progressors. Patients who had more than three ROIs in which normal airways became bronchiectatic within 2 years were thus included into the rapid progression group $(n=21)$; the others were included in the slow progression group $(n=18)$. The two groups were compared with respect to baseline characteristics using Chi-square tests.

SPSS version 13.0 was used for the analyses in this study. Values are shown as median (range) unless otherwise indicated. P-values $<0.05$ (two-tailed) are considered to be statistically significant.

\section{Results}

\section{Study population and CT data}

Forty-three patients ( 18 males) with a median age of 15.3 years (range 9-24 years) were included (Fig. 2). Of those 43 patients, 39 patients had four consecutive CTs and four patients had five consecutive CTs. The median interval between two CTs was 2.0 years (range 0.8-3.9). Baseline characteristics of the study cohort are shown in Table 1.

In the $\mathrm{CT}_{\text {baseline }}$ of the included 43 patients, 367 unique ROIs were identified. In 5/367 (1.4\%) ROIs, bronchiectasis turned into a different category before being scored as

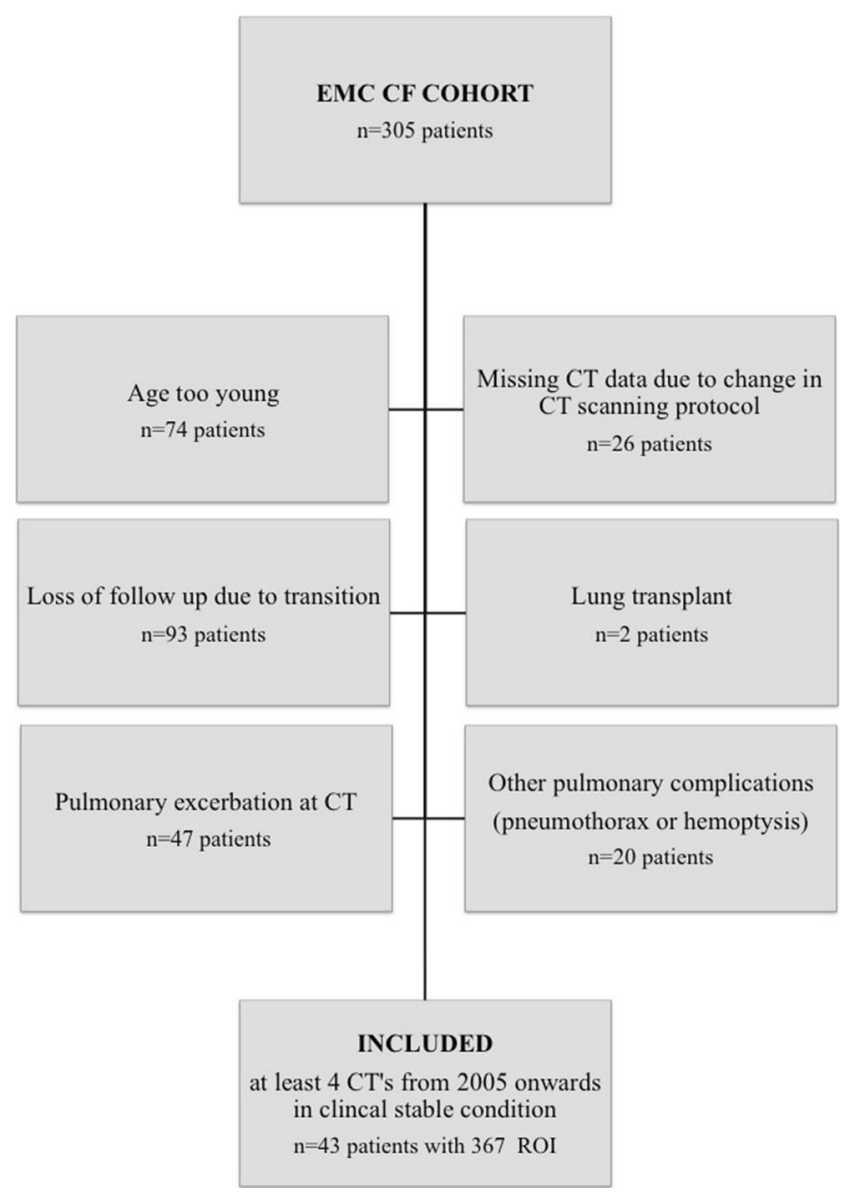

Fig. 2 Flowchart of the study population. $C F$ cystic fibrosis, ROIs regions of interest

bronchiectasis in the baseline scan. In three of the five ROIs the following pathway was identified: bronchiectasis in which the $\mathrm{CT}$ immediately preceding the $\mathrm{CT}$ with bronchiectasis showed normal airways, mucus plugging, bronchiectasis and normal airways. In two of the five ROIs the following pathway was identified: bronchiectasis in which the CT immediately preceding the $\mathrm{CT}$ with bronchiectasis showed atelectasis/ consolidation, bronchiectasisand normal airways.

Previous literature showed that bronchiectasis is irreversible and therefore we excluded those 5/367 cases from further analysis. An overview of the structural lung damage $\left(\mathrm{CT}_{\text {baseline }}-\mathrm{CT}_{\text {minus } 8}\right)$ in our study period is shown in Fig. 3.

The kappa for intra-observer agreement was 0.77 and for interobserver agreement 0.48 . More detailed information about the intra- and interobserver agreement is displayed in Table 1 in the on-line supplementary material.

\section{Bronchiectasis and pre-stages (Fig. 3)}

In 187 of the $362(51.7 \%)$ ROIs bronchiectasis was persistent in all available CTs. For the remaining 175 ROIs in which development of bronchiectasis could be observed, the CT immediately preceding the first $\mathrm{CT}$ with bronchiectasis showed 


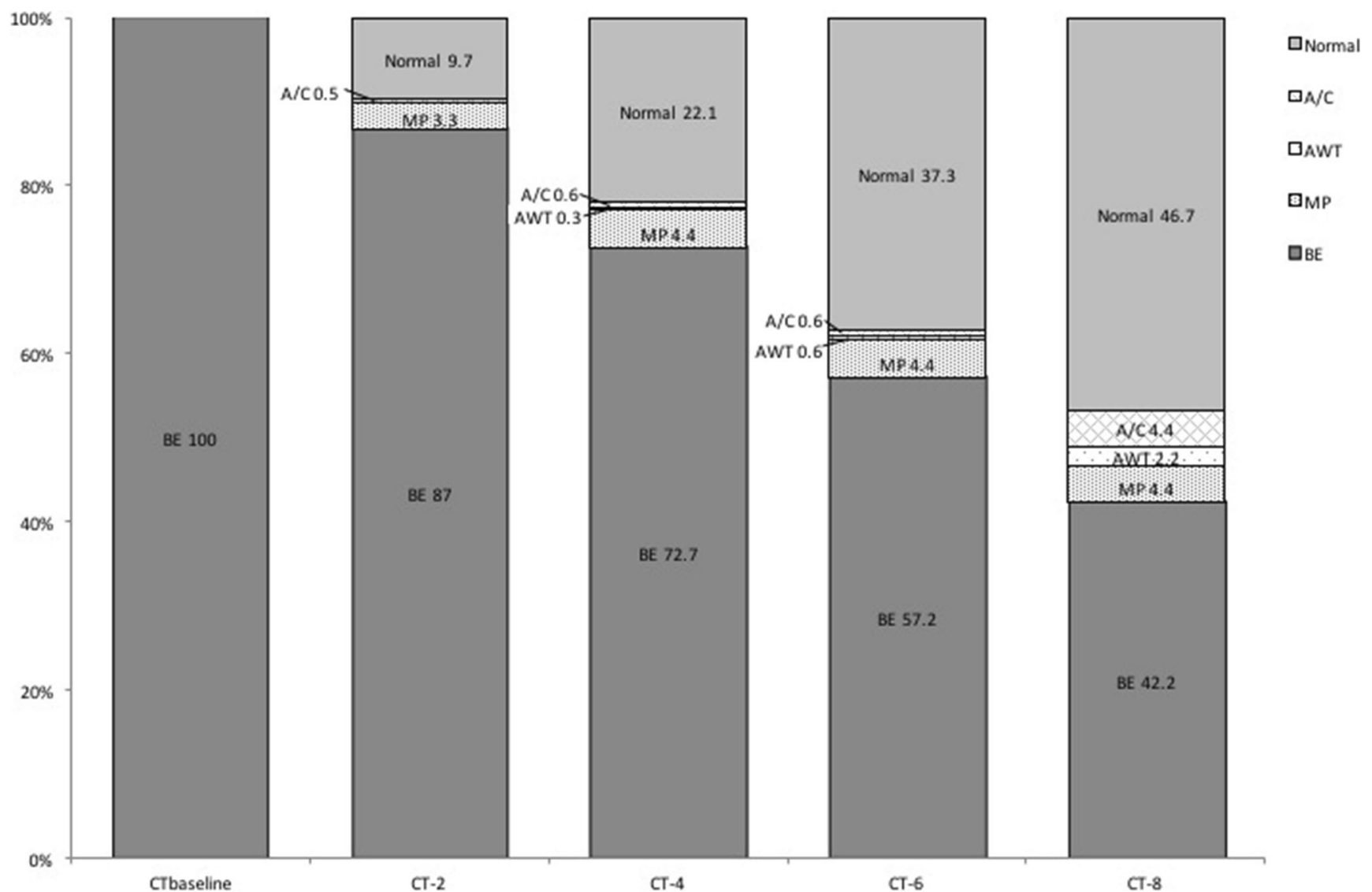

Fig. 3 Structural lung damage over time ( $n=362$ in 43 patients). Dark grey represents bronchiectasis, light-dark grey (area above dark grey) represents mucus plugging, grey dots represent airway wall thickening,

normal airways (139/175, $79.4 \%)$, mucus plugging (31/175, $17.7 \%)$, airway wall thickening $(3 / 175,1.7 \%)$ or atelectasis/ consolidation $(2 / 175,1.1 \%)$.

The most commonly identified pre-stage of bronchiectasis was mucus plugging. In 23 ROIs mucus plugging turned into bronchiectasis within one CT (2 years, range 1.7-2.4). In the other eight ROIs mucus plugging persisted for two CTs (4 years, range 3.7-5.9) before progression to bronchiectasis. Mucus plugging never remained longer than three consecutive CTs, without progressing to bronchiectasis.

\section{Progression to bronchiectasis}

Based on the median number of three ROIs per patient that progressed from normal to bronchiectasis in 2 years, we included 21 patients in the rapid progression group and 18 patients in the slow progression group. There was no statistical difference between these groups with regard to gender $(p=0.47)$, socioeconomic status $(p=0.49)$, genetic defect $(\mathrm{p}=0.78), C F$-related diabetes $(\mathrm{CFRD}, \mathrm{p}=0.16)$, asthma $(\mathrm{p}=0.12)$, allergic bronchopulmonary aspergillosis (ABPA, $\mathrm{p}=0.12$ ) or chronic Pseudomonas aeruginosa infection $(\mathrm{p}=0.39)$. However, there was a borderline significant white dots represent atelectasis/consolidation, light grey (on top) represents normal airways. $\mathrm{CT}_{\text {baseline }}$ is the most recent $\mathrm{CT}$

difference $(\mathrm{p}=0.05)$ for pancreatic insufficiency: all patients included in the rapid group were pancreatic insufficient, while 15 of the 18 patients in the slow progression group were pancreatic insufficient.

\section{Discussion}

This is the first longitudinal study of CT-diagnosed bronchiectasis aiming to identify $\mathrm{CT}$ detectable pre-stages of bronchiectasis in children with CF. We assessed the evolution of uniquely identified ROIs using a deformable image registration technique. Our observations provide a unique insight into the radiological course of developing bronchiectasis.

Our most important findings are that most bronchiectasis appeared within the 2-year timeframe between successive scans. Of the pre-stages that could be identified, mucus plugging was the most common predecessor of bronchiectasis.

Mucus plugging was identified as a potential pre-stage of bronchiectasis. Although no previous studies focused on identifying pre-stages of bronchiectasis have been performed, an earlier study from our group identified mucus plugging as an indicator for bronchiectasis 6 years later [21]. Those results 
suggest a transition from mucus plugging to bronchiectasis, implying that optimising mucociliary clearance treatment is important to prevent bronchiectasis in CF. However, this hypothesis requires further investigation in a prospective $\mathrm{CT}$ study.

Development of bronchiectasis without clearly identifiable pre-stages 2 years earlier was commonly seen in this study. It is possible that the development of most bronchiectasis is an acute process and not caused by a slow continuous progressive transition. Therefore, we hypothesized that there are two possible phenotypes: one for rapid progression of bronchiectasis and one for more slowly developing bronchiectasis.

A sub-group analysis (rapid vs. slow progression) was performed to identify risk factors for the acute development of bronchiectasis in our relatively small single-centre cohort. This sub-group analysis showed only a borderline significant difference in baseline characteristics between the pancreatic status in the two groups. All patients in the rapid progression group were pancreatic insufficient, versus $80 \%$ of the patients in the slow progression group. The importance of pancreatic status as a marker of disease severity has been observed in other studies [6,22]. Although we could not identify an association between severe CFTR genotype and progression of bronchiectasis in our cohort aged 9-24 years, we must acknowledge our small sample size, which may have resulted in inadequate power to detect this association. Previous research by Mott et al. [6] did observe a significant association between genotype and bronchiectasis progression in children below the age of 6 years.

To gain further insight into the pathophysiology of bronchiectasis, it would be interesting to also include trapped air in the analyses, given that an association between trapped air and persistence or progression of bronchiectasis has recently been shown in young children with CF [6]. The most sensitive method to detect trapped air is an expiratory spirometercontrolled CT [23]. For our current study, we did not have sufficient spirometer-controlled expiratory CTs to include this analysis.

There are some limitations to this study. The longitudinal, retrospectively analysed data used in this study were collected from a single centre, which may reduce the generalizability of our results. Data were collected as part of the annual evaluation. During the study period our annual evaluation protocol changed to improve the structure of the follow-up schedule for routine chest CTs so that every child has a CT at the same age $(2,4,6,8,10,12,14,16$ and 18 years) resulting in a larger time range between two CTs for some patients. CTs were performed on different CT scanners, due to the introduction of newer and quicker CT scanners. Furthermore, CT protocols changed during our study period to low-dose CT protocols and spirometer-controlled CT scanning, to reduce radiation and improve image quality [23]. We cannot exclude that the use of these different CT scanners and CT protocols may have reduced the sensitivity to detect pre stages of bronchiectasis. However, it is well recognized that the use of scoring systems is relatively insensitive to differences in CT scanners and CT protocols [24]. Our study is the first to identify ROIs and retrospectively assess the status of these regions in the years preceding bronchiectasis; this scoring strategy has not been used in other studies. Nevertheless, the kappa scores for the intra- and interobserver variability for the sub-scores were above 0.77 and 0.48 , respectively. This suggests that our approach was sufficiently reproducible. Regarding the 301 ROIs with bronchiectasis in $\mathrm{CT}_{\text {baseline, }}$, the two observers scored the same in all but four ROIs, indicating an excellent agreement between observers regarding baseline bronchiectasis. A disadvantage of our scoring method is that we may have missed changes such as subtle airway wall thickening, as scoring is considered a not very sensitive and reproducible method to quantify airway wall thickening [19]; in most CT-scoring studies the kappa for airway wall thickening is low. It is also very possible that when more sensitive automated image analysis tools are developed, subtle changes can be picked up and more pre-stages can be identified.

\section{Clinical implications}

Our study shows the treacherous nature of CF lung disease in a cohort of patients who are receiving standard treatment as in the majority of cases bronchiectasis developed within 2 years without identifiable pre-stages. The only pre-stage that was identified in $17.7 \%$ of cases of bronchiectasis was mucous impaction. Hence, this observation in a patient warrants close attention by the CF team to further improve mucociliary clearance. Furthermore, our results suggest that in some patients the development of bronchiectasis can occur rapidly. The identification of pre-stages of bronchiectasis early on might create a treatment opportunity before irreversible bronchiectasis occurs. Therefore, an annual CT may be considered in children with a rapid progression of bronchiectasis. Nevertheless, additional studies are needed to identify the risk factors leading to the sudden development of bronchiectasis. In future clinical intervention studies are needed that aim to prevent this 'sudden' development of bronchiectasis.

Acknowledgements The authors wish to acknowledge the invaluable input of Pierluigi Ciet, our observers (David Zitter and Hendaye Ozturk), and the members of our lung analysis team (Tim Rosenow and Karla Logie) for critical reading of the manuscript. Furthermore, we acknowledge Gilead Sciences Inc and the Sophia Fund - "steun door Zeevaart" for their unconditional grants. We would also like to acknowledge The Netherlands Organisation for Scientific Research (NWO) for financially supporting Marleen de Bruijne and Vertex for financially supporting Adria Perex Rovira. Harm. A.W.M. Tiddens is heading the 
ErasmusMC image analysis core laboratory LungAnalysis. Furthermore, the authors declare that they have no conflicts of interest.

The scientific guarantor of this publication H.A.W.M. Tiddens, MD, $\mathrm{PhD}$ and the other authors of this manuscript declare no other relationships with any companies whose products or services may be related to the subject matter of the article. One of the authors has significant statistical expertise. Institutional Review Board approval was obtained. Written informed consent was obtained from all subjects (patients) in this study. Methodology: retrospective, observational, performed at one institution.

Open Access This article is distributed under the terms of the Creative Commons Attribution 4.0 International License (http:// creativecommons.org/licenses/by/4.0/), which permits unrestricted use, distribution, and reproduction in any medium, provided you give appropriate credit to the original author(s) and the source, provide a link to the Creative Commons license, and indicate if changes were made.

\section{References}

1. Loeve M, van Hal PT, Robinson P, de Jong PA, Lequin MH, Hop WC et al (2009) The spectrum of structural abnormalities on CT scans from patients with $\mathrm{CF}$ with severe advanced lung disease. Thorax 64:876-882

2. de Jong PA, Nakano Y, Hop WC, Long FR, Coxson HO, Pare PD et al (2005) Changes in airway dimensions on computed tomography scans of children with cystic fibrosis. Am J Respir Crit Care Med 172:218-224

3. de Jong PA, Nakano Y, Lequin MH, Mayo JR, Woods R, Pare PD et al (2004) Progressive damage on high resolution computed tomography despite stable lung function in cystic fibrosis. Eur Respir J 23: 93-97

4. Tiddens HA, Brody AS (2007) Monitoring cystic fibrosis lung disease in clinical trials: is it time for a change? Proc Am Thorac Soc 4: 297-298

5. Barker AF (2002) Bronchiectasis. N Engl J Med 346:1383-1393

6. Mott LS, Park J, Murray CP, Gangell CL, de Klerk NH, Robinson PJ et al (2012) Progression of early structural lung disease in young children with cystic fibrosis assessed using CT. Thorax 67:509-516

7. Sly PD, Brennan S, Gangell C, de Klerk N, Murray C, Mott L et al (2009) Lung disease at diagnosis in infants with cystic fibrosis detected by newborn screening. Am J Respir Crit Care Med 180: 146-152

8. Stick SM, Brennan S, Murray C, Douglas T, von Ungern-Sternberg BS, Garratt LW et al (2009) Bronchiectasis in infants and preschool children diagnosed with cystic fibrosis after newborn screening. J Pediatr 155:623-8.e1

9. Martinez TM, Llapur CJ, Williams TH, Coates C, Gunderman R, Cohen MD et al (2005) High-resolution computed tomography imaging of airway disease in infants with cystic fibrosis. Am J Respir Crit Care Med 172:1133-1138

10. Sly PD, Gangell CL, Chen L, Ware RS, Ranganathan S, Mott LS et al (2013) Risk factors for bronchiectasis in children with cystic fibrosis. N Engl J Med 368:1963-1970

11. Long FR, Williams RS, Castile RG (2004) Structural airway abnormalities in infants and young children with cystic fibrosis. J Pediatr 144:154-161

12. Wainwright CE, Vidmar S, Armstrong DS, Byrnes CA, Carlin JB, Cheney J et al (2011) Effect of bronchoalveolar lavage-directed therapy on Pseudomonas aeruginosa infection and structural lung injury in children with cystic fibrosis: a randomized trial. JAMA 306:163-171

13. de Jong PA, Lindblad A, Rubin L, Hop WC, de Jongste JC, Brink $\mathrm{M}$ et al (2006) Progression of lung disease on computed tomography and pulmonary function tests in children and adults with cystic fibrosis. Thorax 61:80-85

14. Flume PA (2009) Pulmonary complications of cystic fibrosis. Respir Care 54:618-627

15. Loebinger MR, Wells AU, Hansell DM, Chinyanganya N, Devaraj A, Meister M et al (2009) Mortality in bronchiectasis: a long-term study assessing the factors influencing survival. Eur Respir J 34: 843-849

16. Stanojevic S, Wade A, Stocks J, Hankinson J, Coates AL, Pan H et al (2008) Reference ranges for spirometry across all ages: a new approach. Am J Respir Crit Care Med 177:253-260

17. Loeve M, Gerbrandts K, Tiddens HA, Hartmann I, Hop WC (2011) Bronchiectasis and pulmonary exacerbations in children and young adults with Cystic Fibrosis. Chest 140:178-185

18. Brody AS, Kosorok MR, Li Z, Broderick LS, Foster JL, Laxova A et al (2006) Reproducibility of a scoring system for computed tomography scanning in cystic fibrosis. J Thorac Imaging 21:14-21

19. de Jong PA, Tiddens HA (2007) Cystic fibrosis specific computed tomography scoring. Proc Am Thorac Soc 4:338-342

20. Fleiss JL Statistical methods for rates and proportions, 2nd edn. Wiley, New York. ISBN 0-471-26370-2

21. Tepper LA, Utens EM, Caudri D, Bos AC, Gonzalez-Graniel K, Duivenvoorden HJ et al (2013) Impact of bronchiectasis and trapped air on quality of life and exacerbations in cystic fibrosis. Eur Respir J 42:371-379

22. Simanovsky N, Cohen-Cymberknoh M, Shoseyov D, Gileles-Hillel A, Wilschanski M, Kerem E et al (2013) Differences in the pattern of structural abnormalities on CT scan in patients with cystic fibrosis and pancreatic sufficiency or insufficiency. Chest 144:208-214

23. Mott LS, Graniel KG, Park J, de Klerk NH, Sly PD, Murray $\mathrm{CP}$ et al (2013) Assessment of early bronchiectasis in young children with cystic fibrosis is dependent on lung volume. Chest 144:1193-1198

24. Tiddens H, van Straten M, Ciet P (2013) Computed tomography. In: Ernst E, Midulla F, (eds) Pediatric respiratory medicine: the european respiratory society. p 166-76 